\title{
Pressions instationnaires générées par une poche de cavitation partielle
}

\author{
Mohamed Farhat, François Avellan, Francisco Pereira \\ Institut de machines hydrauliques et de mécanique des fluides \\ Ecole Polytechnique Fédérale de Lausanne \\ 33, avenue de Cour, $\mathrm{CH} 1007$ Lausanne
}

\section{Introduction}

En matière d'érosion de cavitation dans les machines hydrauliques, l'une des situations les plus dangereuses est celle qui correspond au développement d'une poche de cavitation à l'entrée de l'aubage [1].

Sur la base de mesures expérimentales effectuées dans le générateur de cavités tourbillonnaires [2], ainsi que de mesures d'érosion conduites dans notre tunnel de cavitation à grande vitesse $[3,4]$, nous avons introduit la notion de puissance érosive [5]. L'objectif de cette étude est de valider cette notion. Pour ce faire, nous nous proposons de mesurer les fluctuations de pression derrière une poche de cavitation partielle simultanément avec ses dimensions. Ces mesures sont effectuées à l'aide d'un profil NACA009 équipé de 30 capteurs piézo-résistifs de pression absolue. Les dimensions de la poche en temps réel sont obtenues grâce à une tranche de lumière pulsée. L'instrumentation est décrite en portant une attention particulière à la synchronisation entre la saisie des pressions et les visualisations. Les résultats obtenus sont analysés dans le cadre des hypothèses qui nous ont conduit à élaborer la notion de puissance érosive [5].

\author{
Moyens expérimentaux
}

\section{Instrumentation de pression}

Les essais ont été réalisés dans la veine d'essai du tunnel de cavitation de l'IMHEF [6]. Le profil utilisé est un profil NACA009 symétrique tronqué à $90 \%$. Trente capteurs KELLER piézo-résistifs de pression absolue sont implantés sur le profil, figures 1 et 2 . D'une étendue de mesure de 0 à 28 bar, ces capteurs ont été détruits par la cavitation et remplacés par des capteurs d'une gamme de mesure de 0 à 200 bar. Chaque capteur est alimenté par une source de courant indépendante et dispose d'un amplificateur et d'un filtre passe-bande séparés. Les amplificateurs utilisés ont un gain ajustable et une fréquence de coupure maximale de $100 \mathrm{kHz}$. L'acquisition des signaux de pression est assurée par deux enregistreurs numériques échantillonnant respectivement 32 canaux à $5 \mathrm{kHz}$ et 4 canaux à $5 \mathrm{MHz}$. Le schéma d'ensemble est reporté figure 3.

L'étalonnage statique des capteurs est effectué le profil monté dans la veine d'essai et en pressurisant le tunnel de 0,2 à 10 bar. Un étalon de pression sert de référence.

\section{Pressure fluctuations downstream a leading edge cavity}

To validate the erosive power model that we have formerly introduced, we intend in this work to compare the erosion tests previously performed on a NACA009 blade with wall pressure fluctuations measured downstream a leading edge cavity. Furthermore, the video acquisition of the main cavity length helps us in finding out the relationship between the vortex shedding frequency and the main cavity pulsation. 


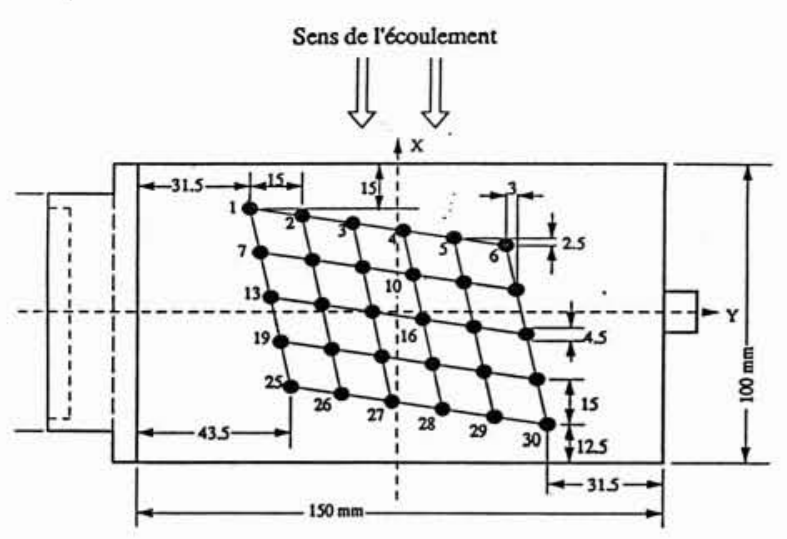

1. Implantation des capteurs sur le profil NACAOO9.

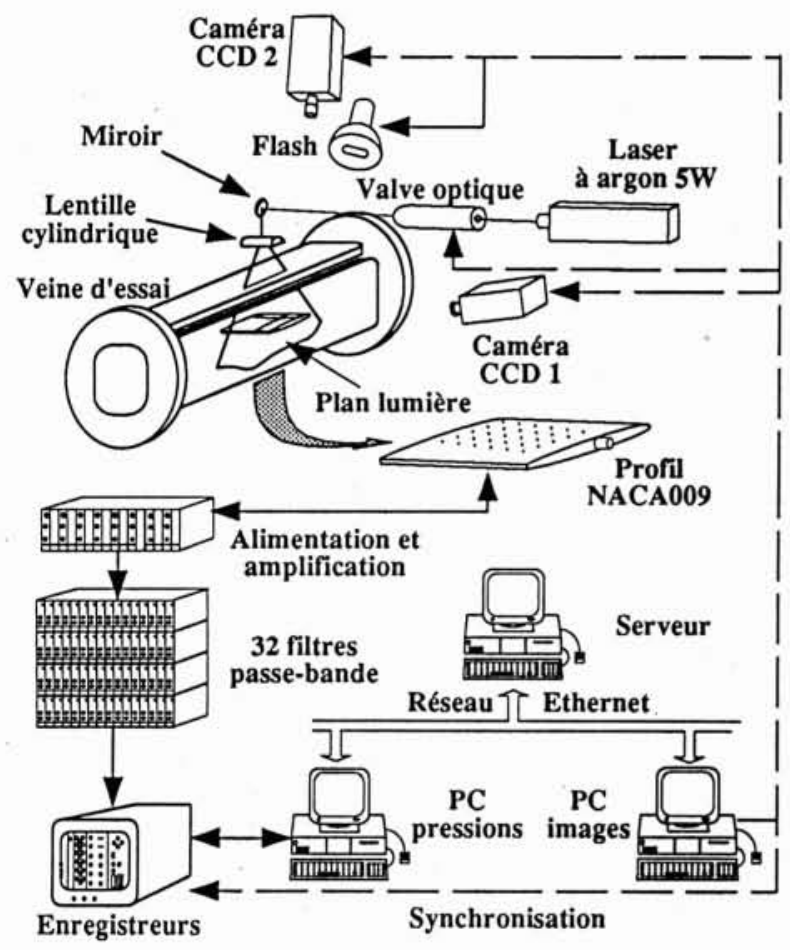

3. Schéma d'ensemble de l'instrumentation.

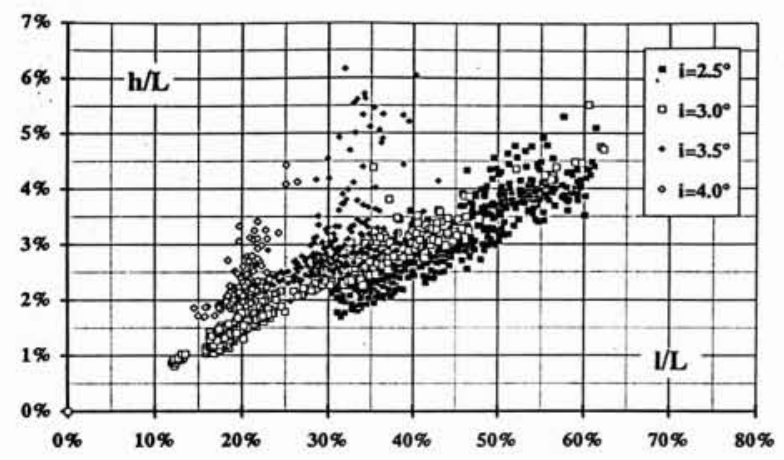

5. Hauteur de la poche en fonction de sa longueur normalisée par la corde.

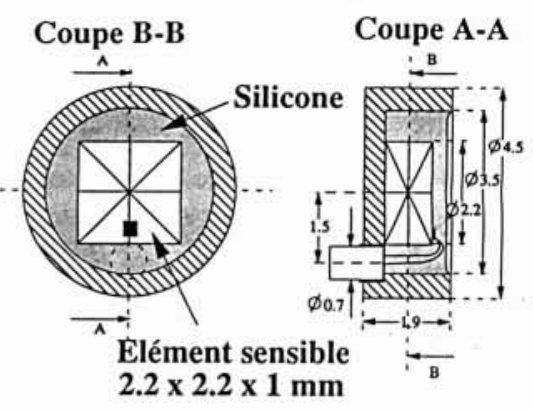

Prise de pression $(0.5 \mathrm{~mm})$

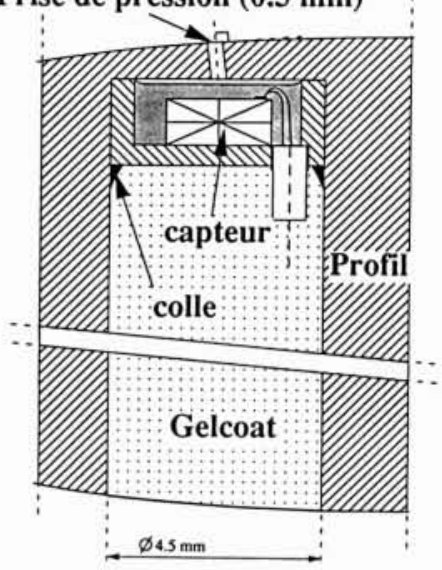

2. Détail et montage d'un capteur.

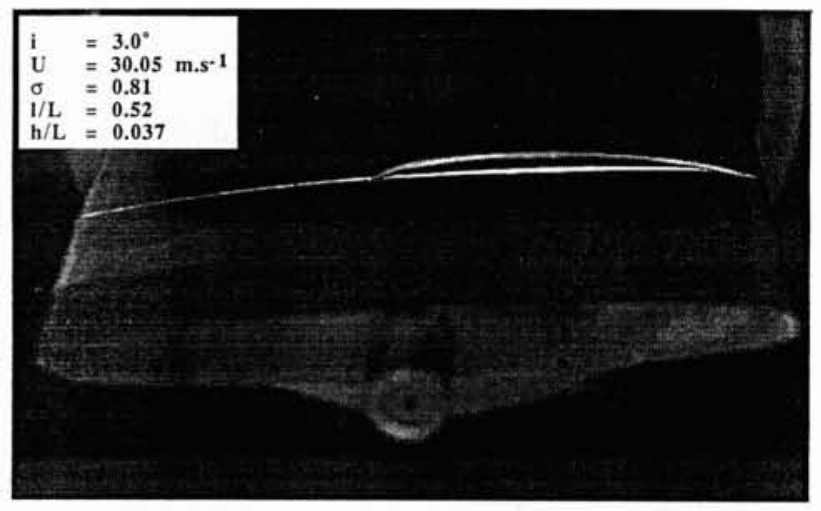

4. Visualisation du contour de la poche par tranche de lumière.

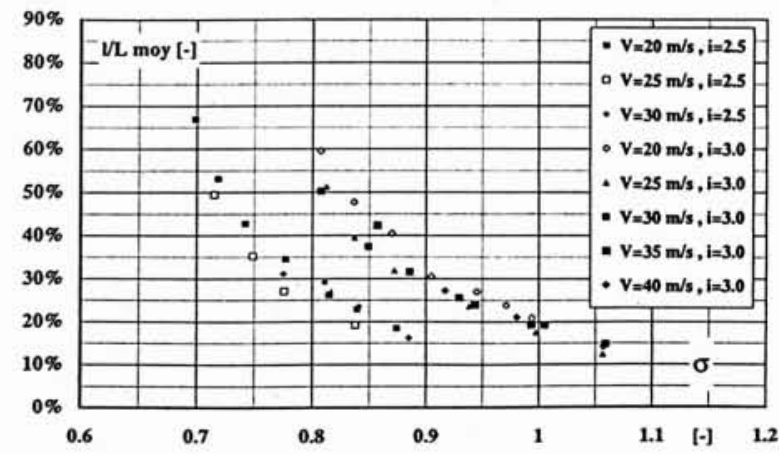

6. Longueur relative moyenne en fonction du cocfficient de cavitation. 
L'étalonnage dynamique est réalisé à l'aide d'une source de pression ponctuelle. Celle-ci est obtenue par la décharge d'un condensateur entre 2 électrodes immergées. La courte durée de cette décharge (environ $10 \mu \mathrm{s}$ ) permet une excitation à large bande. Par ailleurs, un hydrophone B\&K 8103 est utilisé comme capteur de référence. Les fonctions de transfert des différents capteurs ainsi établies mettent en évidence une amplification du signal d'entrée autour de la fréquence de $20 \mathrm{kHz}$. Cette fréquence correspond à la fréquence propre du résonateur de Helmholtz constitué par le trou de prise de pression associé au volume libre du logement du capteur.

\section{Visualisations}

Le profil instantané de la poche de cavitation partielle est relevé à l'aide d'une caméra $\mathrm{CCD}$, la poche étant éclairée par une tranche de lumière laser $[7,8]$. Un obturateur électro-optique permet de générer des impulsions lumineuses d'une durée de $0,5 \mu \mathrm{s}$. Une deuxième caméra $\mathrm{CCD}$ associée à un flash stroboscopique permet l'enregistrement des phénomènes en cours sur magnétoscope, figure 3. L'acquisition se fait à l'aide d'une carte image numérisant le signal vidéo sur 256 niveaux de gris à la cadence de $25 \mathrm{~Hz}$. Cette carte permet, pendant le temps d'acquisition des pressions, de sauvegarder 27 images contiguës. Un exemple en est donné figure 4.

Le contour de la poche est défini en établissant sur chaque ligne verticale d'une image le barycentre des pixels pondérés par le carré de leur intensité lumineuse. Un raccordement entre le profil et ces points affectés d'un écart type est effectué dans les zones de début et de fin de poche par un polynôme de degré inférieur à 5. Les intersections du polynôme avec le profil donnent la longueur projetée sur la corde. L'épaisseur correspond à la différence maximale entre les barycentres et la droite joignant les points d'intersection. Une correction est ensuite apportée pour tenir compte de l'angle de visée.

\section{Protocole de mesure}

4 processus différents doivent être contrôlés simultanément :

- La saisie des paramètres globaux de l'expérience, soient la vitesse amont $U$, l'incidence $i$ du profil et le coefficient de cavitation $\sigma$.

- L'acquisition des 30 signaux de pressions instationnaires.

- La saisie des profils de poche avec la tranche de lumière.

- L'enregistrement des images de cavitation sur magnétoscope.

Un ordinateur, de type PC compatible exploité sous Unix, est dédié à l'acquisition des paramètres globaux de l'écoulement. Connecté au réseau Ethernet, il assure le rôle de serveur d'informations en temps réel. Un deuxième PC est dédié à l'acquisition des signaux de pression. Enfin, un troisième PC spécialisé dans le traitement d'images contrôle les deux derniers processus et la synchronisation de l'ensemble, figure 3.
Pour tenir compte de la dynamique des phénomènes observés, la synchronisation entre acquisition d'image, enregistrements de pression et lecture des paramètres de l'écoulement est essentielle. Pour la réaliser, un ordre est tout d'abord envoyé par le réseau au serveur afin d'activer la lecture des paramètres du tunnel. Les acquisitions du profil de la poche et des pressions sont alors déclenchées par un même signal généré par le PC de contrôle.

L'estimation des valeurs moyennes des trente pressions est effectuée sur la base de 16 lots indépendants de 1024 échantillons chacun. Les signaux sont filtrés à $2 \mathrm{kHz}$ passe-bas et numérisés à une cadence de $5 \mathrm{kHz}$. Par ailleurs, l'analyse spectrale des fluctuations de pression est conduite pour les capteurs $\mathrm{N}^{\text {os }} 4,10$ et 16 . Les densités spectrales de puissance sont alors estimées en moyennant les spectres instantanés sur 32 lots contigus de 4096 échantillons chacun filtrés à $20 \mathrm{kHz}$ et numérisés à une cadence de $50 \mathrm{kHz}$.

\section{Résultats}

\section{Morphologie de la poche de cavitation}

Les contours de poche ont été relevés aux incidences $2,5^{\circ}, 3,0^{\circ}, 3,5^{\circ}$ et $4,0^{\circ}$ et pour des vitesses amont comprises entre 20 et $40 \mathrm{~m} . \mathrm{s}^{-1}$. Le dépouillement de ces contours nous permet de suivre l'évolution de l'épaisseur instantanée $h$ en fonction de la longueur instantanée 1 de la poche de cavitation normalisée par la corde $L$ du profil, figure 5. Elle fait apparaitre une très forte relation linéaire entre ces deux grandeurs pour une incidence donnée. Le rapport $\frac{h}{1}$ est par ailleurs compris entre $7 \%$ et $8 \%$. Toutefois, une dispersion est relevée pour les incidences supérieures à $3,0^{\circ}$. L'évolution de la longueur moyenne $\frac{1}{L}$ en fonction du coefficient de cavitation $\sigma$ est présentée

figure 6. Elle apparaît contradictoire avec le phénomène d'allongement de la poche avec la vitesse moyenne déjà observé dans [7] et confirmé dans [9]. En effet, le couplage hydroélastique observé sur une large gamme de vitesses amont entraîne un bloquage de la longueur de la poche. Cette interaction entre les pulsations de la poche et les vibrations du profil a pu être atténuée en supprimant le couplage hydroélastique par une légère modification du bord de fuite. Nous avons alors retrouvé le phénomène d'allongement de la poche avec le nombre de Reynolds.

\section{Dynamique des cavités érosives}

Le mécanisme de génération des cavités a déjà été décrit par Dupont et al. [5]. Dans la zone de cisaillement intense correspondant à l'interface eau-vapeur, sont générées des lignes de vorticité perpendiculaires à l'écoulement. L'interaction de ces lignes avec les cônes de cavitation crée des instabilités de type Kelvin-Helmholtz qui conduisent à la formation de cavités tourbillonnaires en forme de $U$ inversé analogues à celles observées par SASAKI et KIYA [11] derrière une bulle de décollement. Ces cavités viennent ensuite s'effondrer à l'aval de la 

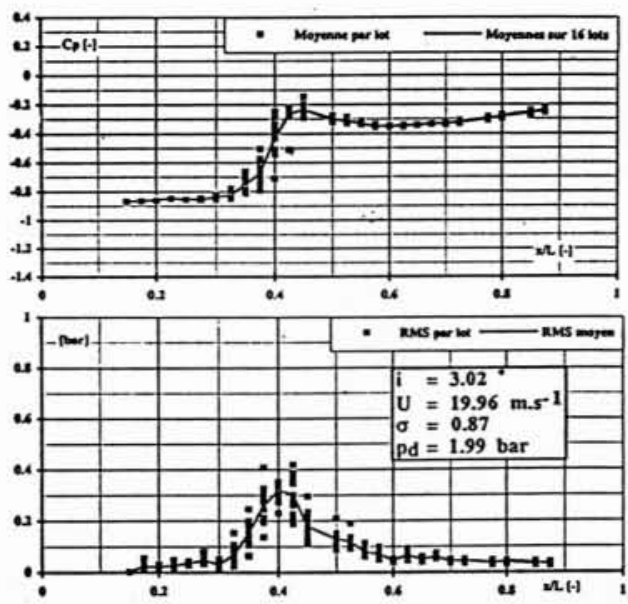

7. Coefficient de pression, écart types de la pression et contour de poche correspondant.

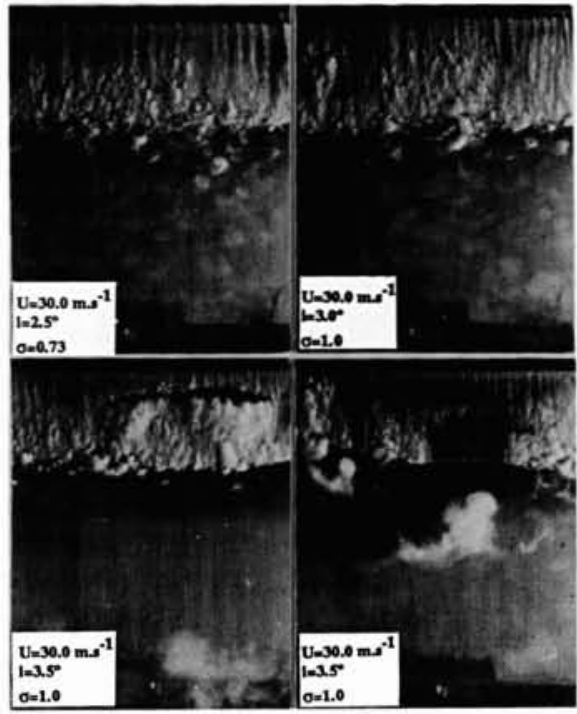

8. Photographies de la poche en vue de dessus.

poche de cavitation provoquant des fluctuations de pression intenses.

Les distributions des valeurs moyennes et des écarts types des pressions sont représentées figure 7 ainsi que le contour de poche associé. Il en résulte que le maximum des fluctuations de pression a lieu dans la zone de fermeture de la poche. Ce résultat est en accord avec les mesures d'érosion déjà effectuées sur ce profil [4] et selon lesquelles le maximum de l'érosion se situait dans cette même zone. Par conséquent, les fluctuations de pression sont représentatives de l'intensité des implosions répétées des cavités.

L'examen des visualisations photographiques dont un exemple est donné figure 8 ainsi que les visualisations cinématographiques ultra-rapides (10 000 images/ seconde) fait apparaître deux aspects distincts dans la génération des cavités érosives confirmé dans $[9,10,12$, 13]. Ainsi, pour les incidences inférieures à $3^{\circ}$, la poche

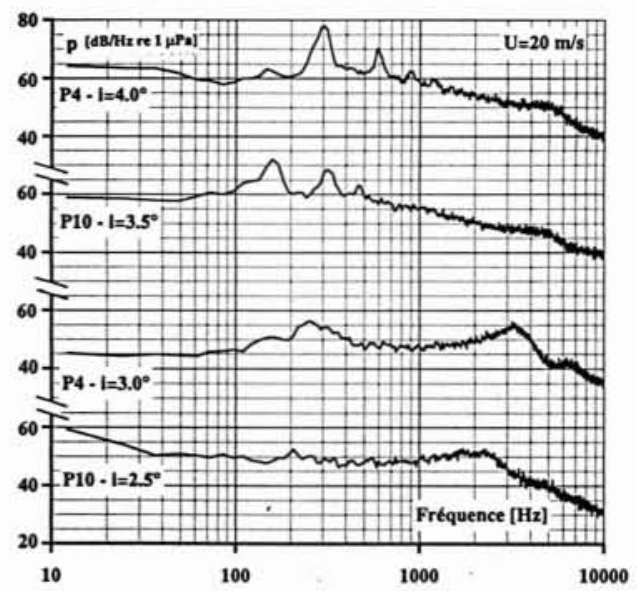

9. Influence de l'incidence sur les densités spectrales de puissance de la pression.

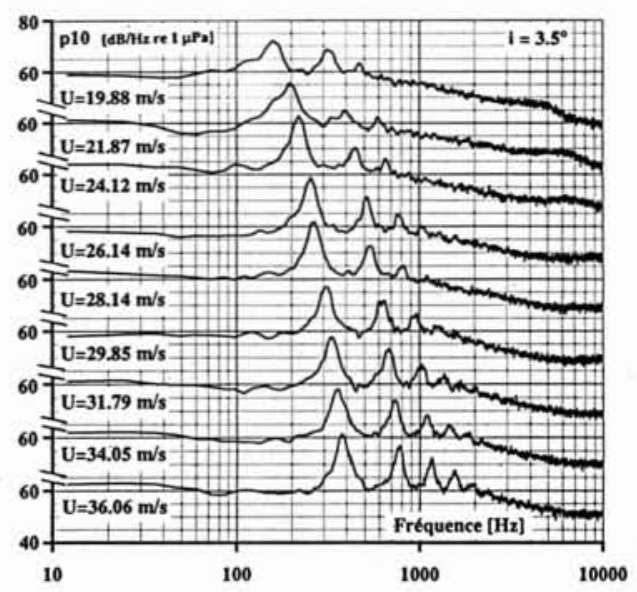

10. Influence de la vitesse amont sur les densités spectrales de puissance de la pression. de cavitation est relativement stable et nous assistons à la génération de structures de petite taille. Par contre, aux incidences supérieures, la poche devient instable et des structures de taille plus importante sont générées en se détachant près du bord d'attaque (figure 8 ). Ceci est attribué à un couplage entre les pulsations de la poche et le lâcher des structures érosives. Il a été remarqué que pour une incidence donnée, ce couplage est favorisé lorsque la vitesse de l'écoulement ou, indépendamment, la longueur de la poche est augmentée. Ces deux aspects se retrouvent sur les spectres de puissance de la pression dans la zone de fermeture de la poche établis pour une vitesse amont de $20 \mathrm{~m} \cdot \mathrm{s}^{-1}$ et pour les incidences $2,5^{\circ}$, $3,0^{\circ}, 3,5^{\circ}$ et $4,0^{\circ}$, figure 9 . Dans le premier cas, les spectres de puissance ne font apparaître aucune fréquence privilégiée. Dans le second cas, le couplage entre les pulsations de la poche et la génération des tourbillons donne lieu à une régularité dans le lâcher des cavités 
érosives mise en évidence sur les spectres de puissance. Dans la présente étude, nous nous sommes restreints à étudier la dynamique de la poche de cavitation en présence de ce couplage du fait de son importance en matière d'érosion de cavitation $[4,12]$.

Les spectres de puissance de la pression $p_{10}$ représentés figure 10 sont établis pour différentes valeurs de la vitesse amont et pour une incidence de $3,5^{\circ}$. Le coefficient de cavitation est ajusté pour faire coöncider la zone de fermeture de la poche avec la position du capteur $\mathrm{N}^{\bullet} 10$. Ces spectres mettent en évidence une fréquence principale qui croît avec la vitesse amont. Cette fréquence est naturellement attribuée aux pulsations de la poche.

Les spectres de puissance relatifs aux pressions $p_{4}, p_{10}$ et $p_{16}$ présentés figure 11 ont été établis pour une vitesse amont de $28 \mathrm{~m} \cdot \mathrm{s}^{-1}$ et une incidence de $4,0^{\circ}$. La zone de fermeture de la poche coïncide avec la position du capteur $\mathrm{N}^{\circ} 4$. Nous retrouvons sur les 3 spectres la même fréquence principale. Compte tenu des positions éloignées des capteurs 10 et 16 par rapport à la zone de fermeture de la poche, le pic de puissance observé à cette fréquence correspond au passage des cavités tourbillonnaires. Ce résultat est confirmé par les fonctions de cohérence représentées sur la même figure. Il en résulte que le lâcher de ces structures est fortement modulé par les fluctuations de la poche.
Nous avons représenté figure 12 l'évolution de la fréquence $f$ de lâcher des cavités en fonction du rapport de la vitesse amont et de la longueur de la poche. Nous observons que le nombre de Strouhal St défini par:

$$
\mathrm{St}=\frac{f \cdot l}{U}
$$

demeure constant lorsque la vitesse amont et la longueur de la poche varient. Par conséquent, ce nombre constitue un paramètre représentatif du taux de génération des cavités érosives. Ce résultat confirme l'hypothèse avancée dans [5] qui établissait la notion de puissance érosive.

Les fonctions d'intercorrélation présentées figure 11 et relatives aux pressions $p_{4}, p_{10}$ et $p_{16}$ permettent, à partir des retards entre les différents signaux, d'estimer la vitesse de convection des cavités érosives à l'aval de la poche de cavitation. La figure 13 montre l'évolution de cette vitesse calculée entre les positions des 3 capteurs en fonction de la vitesse amont. Outre l'allure naturellement croissante de ces courbes, nous remarquons une augmentation de la vitesse de convection des cavités érosives entre les capteurs 4 et 16 . Nous avons représenté sur le même graphe l'évolution analogue de la vitesse en paroi $U_{p}$ déduite de la pression moyenne par la relation de Bernoulli :

$$
U_{p} \approx U \sqrt{1-C_{p}} .
$$
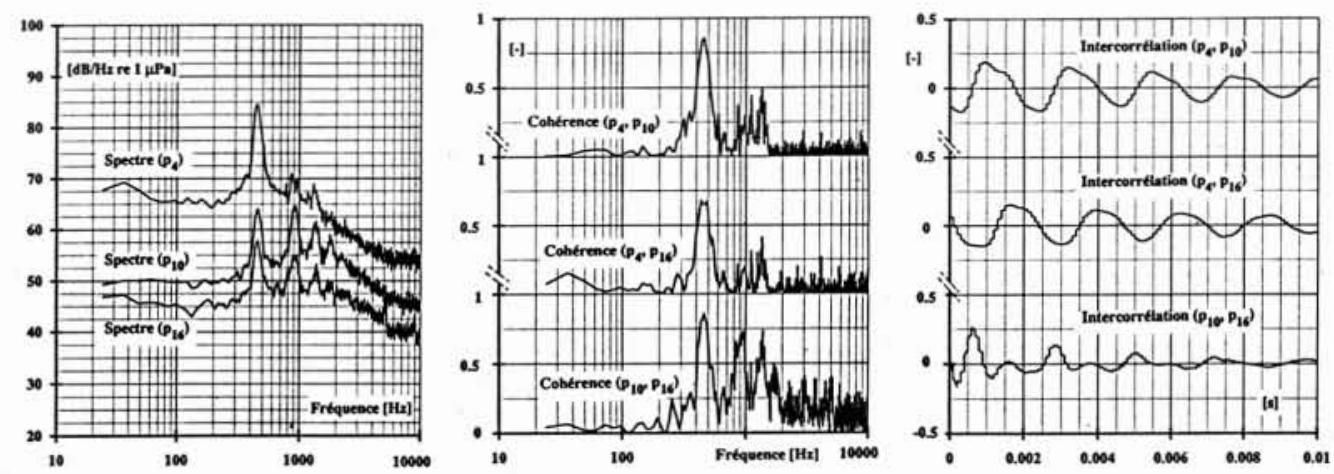

11. Spectres de puissance, fonctions de cohérence et d'intercorrélation $; U=27,84 \mathrm{~m} \cdot \mathrm{s}^{-1}, \sigma=1,34, i=4,07^{\circ}$.

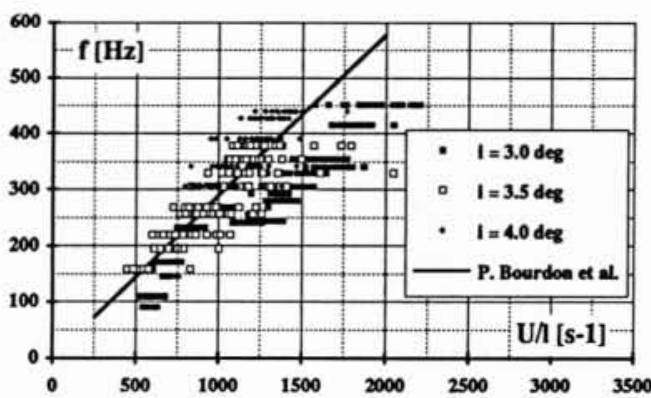

12. Fréquence de lâcher des cavités érosives en fonction de $\mathrm{U} / l$.

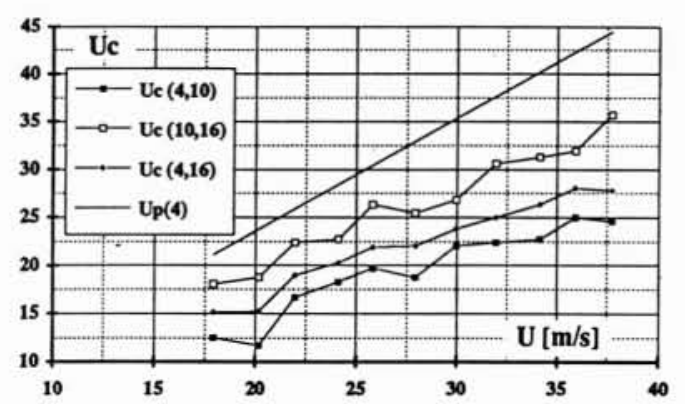

13. Vitesse de convection en fonction de la vitesse amont. 


\section{Puissance érosive}

Sur la base de mesures de pression générée par l'implosion de tourbillons que nous avons entreprises dans le générateur de cavités tourbillonnaires [2], nous avons établi que l'énergie développée lors de cette implosion est proportionnelle à l'énergie potentielle $E$ de la cavité correspondant à son volume maximal $V$ :

$$
E=\left(p_{\text {paroi }}-p_{v}\right) \cdot V
$$

où $p_{\text {paroi }}$ désigne la pression en paroi de la veine en régime permanent.

Dans le cas de l'écoulement autour du profil NACA, l'énergie potentielle d'une cavité érosive prend la forme suivante :

$$
E=\left(p_{\max }-p_{v}\right) \cdot V_{c}
$$

où $V_{c}$ désigne le volume de la cavité et $p_{\max }$ la recompression subie par la cavité et responsable de l'implosion.

$f_{c}$ désignant la fréquence de lâcher des structures érosives, la puissance érosive devient :

$$
\dot{E}=\left(p_{\max }-p_{v}\right) f_{c} V_{c} .
$$

Les dimensions des cavités érosives n'étant pas disponibles, leur échelle de longueur est supposée être représentée par la longueur $l$ de la poche. Ceci constitue l'hypothèse $\mathrm{H1}$ :

$$
V_{c} \approx l^{3} \approx h^{3} .
$$

D'autre part, nous avons montré que le nombre de Strouhal constituait un paramètre représentatif du taux de génération des cavités érosives. Ceci représente l'hypothèse $\mathrm{H} 2$ :

$$
f_{c} \approx f \approx \mathrm{St} \frac{U}{l}
$$

A partir de ces deux hypothèses et en introduisant la normalisation utilisée dans cette étude, la puissance érosive peut être estimée par la relation :

$$
\dot{E}=\frac{1}{2} K \rho\left(C p_{\max }+\sigma\right) S t U^{3} l^{2} \quad K \text { constante } .
$$

Ces deux hypothèses étant confirmées à la fois par les visualisations par tranche de lumière, hypothèse $\mathrm{H} 1$, et par les spectres de fluctuations de pression, hypothèse $\mathrm{H} 2$, il reste à vérifier que ce terme est bien significatif des fortes fluctuations de pressions que subit le profil. Pour ce faire, nous avons d'abord normalisé la puissance érosive avec la valeur $\dot{E}_{0}$ qu'elle prend pour une vitesse $U=20 \mathrm{~m} \cdot \mathrm{s}^{-1}$, une incidence $i=4,0^{\circ}$ et une longueur de poche $l \approx 20 \mathrm{~mm}$. C'est ainsi que nous avons représenté figure 14 les écart types maxima des pressions $p_{4}, p_{10}$ et $p_{16}$ en fonction de la puissance érosive pour deux valeurs de l'incidence. La dépendance linéaire mise en évidence nous permet d'affirmer que cette notion purement hydrodynamique de puissance érosive représente bien l'agressivité des cavités associées à une poche de cavitation partielle. En outre, elle offre la possibilité de transposer cette puissance d'une échelle à une autre ce qui est d'une très grande importance pour la conception et

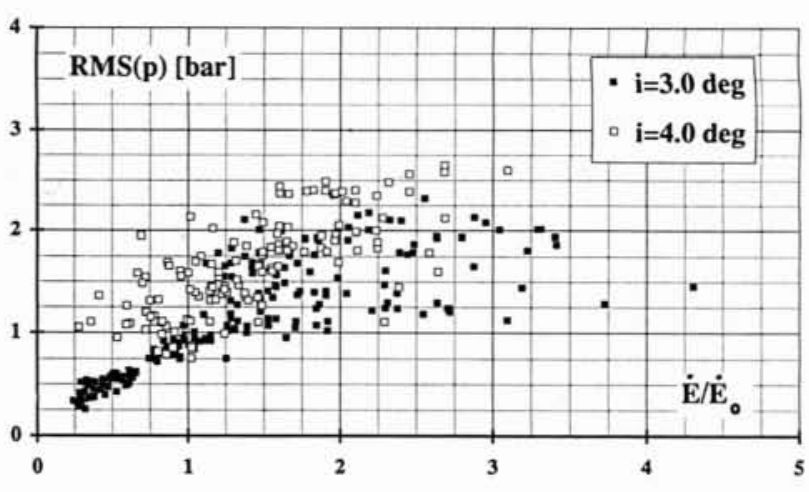

14. Ecart types des fluctuations de pression en fonction de la puissance érosive.

l'exploitation des machines hydrauliques. En effet, il devient envisageable de mesurer sur un modèle, par un moyen indirect, un paramètre significatif des fluctuations de pressions auxquelles la machine est soumise. Ce paramètre peut être un niveau d'accélération ou un niveau de bruit associé à une mesure de longueur de poche. Il serait alors possible de transposer ce niveau à l'échelle du prototype afin de prédire l'érosion de cavitation.

\section{Conclusion}

Grâce à la mise en œuvre simultanée des mesures de pressions instationnaires et des visualisations de poche de cavitation, nous avons pu confirmer les hypothèses qui président à l'élaboration de la notion de puissance érosive associée au développement d'une poche de cavitation de bord d'attaque observé lors des fonctionnements non nominaux des pompes ou des turbines.

La mesure des pressions instationnaires derrière une poche de cavitation partielle montre que lorsque le taux de génération des cavités érosives avoisine la fréquence de pulsation de la poche de cavitation, un couplage apparaît entre ces deux processus. Dans ce cas le lâcher des tourbillons est fortement modulé par la pulsation de la poche. Le nombre de Strouhal bâti sur la longueur de la poche ne dépend que de l'incidence de l'écoulement. En outre, la relation linéaire entre épaisseur et longueur d'une poche laisse à penser que les dimensions des cavités érosives sont aussi déterminées par la longueur de la poche. C'est sur la base de ces résultats que nous avons pu montrer que le niveau des fluctuations de pression varie linéairement avec la puissance érosive.

\section{Remerciements}

Cette étude réalisée au sein du groupe Cavitation de l'IMHEF a été financée par le Nationaler Energie Forschungs Fonds, NEFF et la Direction des Etudes et Recherches de l'Electricité de France, EDF. Nous tenons à remercier Monsieur Louis Bezençon qui a mené à bien la réalisation du profil d'essai et l'implantation des capteurs de pression. 


\section{Nomenclature}

\section{Grandeurs}

$\begin{array}{ll}L & \mathrm{~m} \\ U & \mathrm{~m} \cdot \mathrm{s}^{-1} \\ U_{p} & \mathrm{~m} \cdot \mathrm{s}^{-1} \\ V_{c} & \mathrm{~m}^{3} \\ \dot{E} & \mathrm{~W}\end{array}$

Corde du profil

Vitesse à l'entrée de la veine d'essai Vitesse à la paroi

Volume d'une cavité tourbillonnaire Puissance érosive $\frac{1}{2} \rho\left(C p_{\max }+\sigma\right) S t U^{3} l^{2}$

$C p_{k} \quad-\quad$ Coefficient de pression relatif au capteur $n^{*} k$

$$
\frac{p_{\lambda}-p_{\text {ref }}}{\frac{1}{2} \rho U^{2}}
$$

$\begin{array}{ll}f & \mathrm{~Hz} \\ h & \mathrm{~m} \\ i & \circ \\ l & m \\ p_{k} & \mathrm{~Pa}\end{array}$

Fréquence de pulsation de la poche incidence du profil

Longueur de la poche de cavitation

\begin{tabular}{|c|c|c|}
\hline$p_{\max }$ & $\mathrm{Pa}$ & $\begin{array}{l}\text { Pression maximale sur l'extrados } \\
\text { du profil }\end{array}$ \\
\hline$p_{\text {ref }}$ & $\mathrm{Pa}$ & $\begin{array}{l}\text { Pression statique à l'entrée de la } \\
\text { veine d'essai }\end{array}$ \\
\hline$p_{\mathrm{r}}$ & $\mathrm{Pa}$ & Pression de vapeur \\
\hline$R M S\left(p_{k}\right)$ & $\mathrm{Pa}$ & Ecart type de la pression $p_{k}$ \\
\hline$\rho$ & $\mathrm{kg} \cdot \mathrm{m}^{-3}$ & Masse volumique de l'eau d'essai \\
\hline$\nu$ & $\mathrm{m}^{2} \cdot \mathrm{s}^{-2}$ & Viscosité cinématique de l'eau d'essai \\
\hline
\end{tabular}

Pression moyenne mesurée par le

\section{Nombres adimensionnels}

$C p$

Coefficient de pression $\frac{p-p_{\text {ref }}}{\frac{1}{2} \rho U^{2}}$

$C p_{\max }$

$C p_{k}$

Coefficient de pression maximal

$\mathrm{Re}$

Coefficient de pression mesurée par le capteur $\mathrm{n}^{\circ} k$ Nombre de Reynolds $\frac{U l}{v}$

St

Nombre de Strouhal $\frac{f l}{U}$ capteur $\mathrm{n}^{\circ} k$

\section{Références}

[1] Avellan F., Dupont Ph., Rhyming I.L. - " Generation mechanism and dynamics of cavitation vortices downstream of a fixed leading edge cavity ", Proc. of 17th Symposium on Naval Hydrodynamics, The Hague (The Netherlands), August 29-September 2, 1988, Sessions V, pp. 1-13.

[2] Avellan F., Farhat M. - « Shock pressure generated by cavitation vortex collapse ", Proc. of International Symposium on Cavitation Noise and Erosion in Fluid Systems, ASME Winter annual meeting, San Francisco (USA), December 1989, FED, vol. 88, pp. 119-125.

[3] Bourdon P., Simoneau R., Avellan F., Farhat M. «Vibratory characteristics of erosive cavitation vortices downstream of a fixed leading edge cavity ", Proc. 15th IARH Symposium, September 11-14, 1990, Belgrade (Yugoslavia).

[4] Simoneau R., Avellan F., Kuhn de Chizelle Y. - « On line measurement of cavitation erosion rate on a 2D NACA profile », Proc. of Third International Symposium on Cavitation Noise and Erosion in Fluid System, ASME, December 1989, San Francisco (USA).

[5] Avellan F., Dupont Ph., Farhat M. - "Cavitation erosion power ", Proc. of International Symposium on Cavitation and Multiphase Flow, ASME-JSME meeting, Portland (USA), June 1991, FED, vol. 116, pp. 135-140.

[6] Avellan F., Henry P., Rhyming I.L. - « A new high speed tunnel for cavitation studies in hydraulic machinery ", Proc. of International Symposium on Cavitation Research facilities and techniques », ASME winter annual meeting, Boston (USA), December 13-18, 1991, FED, vol. 88, pp. 95-102.
[7] Dupont Ph., Avellan F. - « Numerical Computation of a leading edge cavity ", Proc. of International Symposium on Cavitation and Multiphase Flow, ASME-JSME meeting, Portland (USA), June 1991, FED, vol. 116, pp. 47-54.

[8] Kueny J.L., Reboud J.L. - « Analysis of partial cavitation : Image processing and numerical prediction », Proc. of International Symposium on Cavitation and Multiphase Flow, ASME-JSME meeting, Portland (USA), June 1991, FED, vol. 116, pp. 55-60.

[9] Joussellin F., Delannoy Y. - « Experimental investigations on unsteady attached cavities ", Proc. of International Symposium on Cavitation and Multiphase Flow, ASMEJSME meeting, Portland (USA), June 1991, FED, vol. 116, pp. 61-66.

[10] Kubota S., Kato H., Yamaguch Y., Maeda M. " Unsteady structure measurement of cloud cavitation on a foil section using conditional sampling technique ».

[11] KyURo SASAKI, MASARU KiYa. - « Three-dimensional Vortex Structure in a leading edge Separation bubble at moderate Reynolds Numbers ", Journal of Fluid Dynamics, September 1992, vol. 113, pp. 405-410.

[12] Kato H., Ye Y.P., Maeda M. - "Cavitation Erosion and Noise Study on a Foil Section ", Proc. of ASME winter meeting, San Francisco (USA), November 1991, FED, vol. 88 , pp. 79-88.

[13] Quang L., Franc J.P., Michel J.M. - « Pressure Pulse Distribution Around Cavity Closure ", Proc. of ASME winter meeting, San Francisco (USA), November 1991, FED, vol. 88, pp. 111-118.

[14] FRY S.A. - « The Damage Capacity of Cavitating Flow from Pulse Height Analysis ". Transactions of the ASME, December 1989, vol. 111, pp. 502-509. 